Post-print of journal article: A. Del Nevo, P. Arena, G. Caruso, P. Chiovaro, P. Di Maio, M. Eboli, F. Edemetti, N. Forgione, R. Forte, A. Froio, et al., Recent progress in developing a feasible and integrated conceptual design of the WCLL BB in EUROfusion project, Fusion Engineering and Design (2019). Doi: 10.1016/j.fusengdes.2019. 03.040 


\title{
Recent progress in developing a feasible and integrated conceptual design of the WCLL BB in EUROfusion Project
}

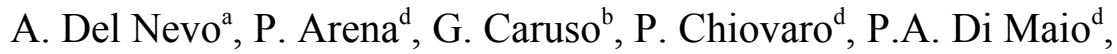 \\ M. Eboli ${ }^{\mathrm{c}}$, F. Edemetti ${ }^{\mathrm{b}}$, N. Forgione ${ }^{\mathrm{c}}$, R. Forte ${ }^{\mathrm{d}}$, A. Froio ${ }^{\mathrm{g}}$, F. Giannetti ${ }^{\mathrm{b}}$, G. Di Gironimo ${ }^{\mathrm{e}}$, \\ K. Jiang ${ }^{h}$, S. Liu ${ }^{h}$, F. Moro ${ }^{f}$, R. Mozzillo ${ }^{\mathrm{e}}$, L. Savoldi ${ }^{\mathrm{g}}$, A. Tarallo ${ }^{\mathrm{e}}$, M. Tarantino ${ }^{\mathrm{a}}$,

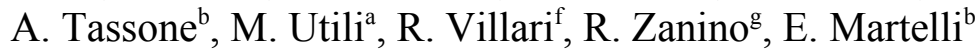 \\ ${ }^{a}$ ENEA FSN-ING, CR Brasimone, Località Brasimone, 40032, Camugnano (BO), Italy \\ ${ }^{b}$ DIAEE, Sapienza University of Rome, Corso Vittorio Emanuele II, 244, 00186, Roma, Italy \\ ${ }^{c}$ DICI, University of Pisa, Largo Lucio Lazzarino 2, 56122, Pisa, Italy \\ ${ }^{d}$ DEIM, University of Palermo, Viale delle Scienze, Edificio 6, 90128, Palermo, Italy \\ ${ }^{e}$ CREATE, University of Naples Federico II, DII, P.le Tecchio 80, 80125, Napoli, Italy \\ ${ }^{f}$ ENEA FSN-FUSTEC-TEN, C.R. Frascati, via E. Fermi 45, 00044 Frascati, Italy \\ ${ }^{g}$ NEMO group, Dipartimento Energia, Politecnico di Torino, 10129 Torino, Italy \\ ${ }^{h}$ Institute of Plasma Physics, Chinese Academy of Sciences, Hefei, Anhui, 230031, China
}

\begin{abstract}
The water-cooled lithium-lead breeding blanket is in the pre-conceptual design phase. It is a candidate option for European DEMO nuclear fusion reactor. This breeding blanket concept relies on the liquid lithium-lead as breeder-multiplier, pressurized water as coolant and EUROFER as structural material. Current design is based on DEMO 2017 specifications. Two separate water systems are in charge of cooling the first wall and the breeding zone: thermo-dynamic cycle is $295-328^{\circ} \mathrm{C}$ at $15.5 \mathrm{MPa}$. The breeder enters and exits from the breeding zone at $330^{\circ} \mathrm{C}$. Cornerstones of the design are the single module segment approach and the water manifold between the breeding blanket box and the back supporting structure. This plate with a thickness of $100 \mathrm{~mm}$ supports the breeding blanket and is attached to the vacuum vessel. It is in charge to withstand the loads due to normal operation and selected postulated initiating events. Rationale and progresses of the design are presented and substantiated by engineering evaluations and analyses. Water and lithium lead manifolds are designed and integrated with the two consistent primary heat transport systems, based on a reliable pressurized water reactor operating experience, and six lithium lead systems. Open issues, areas of research and development needs are finally pointed out.
\end{abstract}

Keywords: WCLL, Breeding Blanket, DEMO, EUROfusion.

\section{Introductory remarks}

The Breeding Blanket (BB) [1] is a key component for the future deployment of nuclear fusion electricity. This component, bounding large part of the plasma chamber, shall ensure to accomplish three main functions: sufficient shielding performances, a reliable tritium (T) production and an efficient heat extraction for electricity production. The Water-Cooled Lithium-Lead (WCLL) BB relies on pressurized water as coolant and Lithium-Lead (PbLi) enriched at $90 \%$ in ${ }^{6} \mathrm{Li}$ as breeder, neutron multiplier and $\mathrm{T}$ carrier. Current design (2018), is based on DEMO 2017[2] specifications, derives from the outcomes of the design studies[3],[4],[5] and R\&D activities [6] conducted in the framework of the EUROfusion Programme [7].

DEMO power plant is a pulsed machine with a burn time of two hours (power pulse) and a dwell time of 10 minutes, because of central solenoid recharge time and vacuum pump performances. This implies that the Power Conversion System (PCS) includes the Energy Storage System (ESS) with HITEC molten salt as secondary fluid, ensuring that the turbine works continuously during the dwell time [8]. The average Neutron Wall Load is 1.05 $\mathrm{MW} / \mathrm{m}^{2}$ and radial nuclear power densities in materials are calculated by MCNP5 code on the basis of the data available (i.e. DEMO2015) [9]. The thermal power deposited in the First Wall (FW) during pulse [10] is due to the heat flux (i.e. thermal radiation, mainly, and the charged particles) and the volumetric heat deposition, both variables along the poloidal profile. Details on requirements and assumptions of DEMO and the breeding blanket are provided in Ref. [2].

\section{Description of WCLL BB 2018}

The design is based on DEMO2017 baseline [11], subdivided into 16 sectors with two inboard and three outboard segments each. Compared with the former design, the space allocated for the BB has been reduced of $300 \mathrm{~mm}$ in radial direction. Following the preliminary studies done in 2016 [12], the WCLL BB relies on the Single Module Segment (SMS) approach, with a breeding element repeated along the poloidal direction. The rationale behind this choice is based on the considerations that the SMS

allows more design options [13]-[17], including the removal of the PbLi manifold and its integration in the Breeding Zone (BZ), thus enhancing the compactness in radial direction;

- maximizes the TBR performances [9], because the continuity of the structures and the reduction of the steel of the caps; 
- eliminates the neutron streaming between the modules, thus enhancing shielding performances;

- facilitates the PbLi fill and drain procedures;

$\square$ may allow the removal of He bubbles if formed in the $\mathrm{BZ}$ and not dissolved in the PbLi [6], [18].

\subsection{The manifold region}

The segment is attached with the vacuum vessel through the Back Supporting Structure (BSS). This is a plate $100 \mathrm{~mm}$ thick (Fig. 1), which is the main structural support in charge of ensuring, under postulated load conditions, thermo-mechanical performances compliant with the rules prescribed by the safety codes. Structural analyses of the segment[12], [19] and sector are carried out with different load combinations: gravity, thermal and electro-magnetic [20]. The combination of the former two loads represents the Normal Operation conditions. Differently, the combination of the three loads, represents the occurrence of the postulated Central Major Disruption (CMD) event. Both load conditions have been verified with the Level A criteria in SDC-IC code using Abaqus FEM code.

Considering the volumetric power density deposited due to neutron flux, it was evaluated that, on the opposite of the outboard, the inboard BSS requires the availability of a coolant system[4]. This analysis was a further motivation to integrate the water manifolds between the BSS and the PbLi manifolds. The water manifolds are connected with the integrated First Wall (FW) and the BZ coolant systems. Coolant flows at $295^{\circ} \mathrm{C}$ in the inlet manifolds, whereas $328^{\circ} \mathrm{C}$ is the temperature at the outlet. The integrated water manifolds are designed to accomplish the following main functions.

$\square$ To be connected with a coolant system capable to ensure a symmetric thermal field in the $\mathrm{BZ}$ and FW[16]. Moreover, because the different lengths of the Double Wall Tubes (DWT), "high" and "low" pressure inlet manifold zones are foreseen to provide an optimal flow distribution in the tubes.

$\square$ To ensure that the structural material in the inboard segment manifold zone (i.e. BSS) are kept at low temperature (i.e. $<350^{\circ} \mathrm{C}$ ).

$\square \quad$ To maximize the shielding performances of the BB zone[9], removing the presence of any undesired void space.

The PbLi manifolds are placed between the water coolant manifold and breeding zones. It is based on a coaxial square structure. The structural function is in charge of the outer EUROFER plates, whereas the inner sheets are used to separate the inlet (outer) from the outlet (inner) manifolds. PbLi temperature is uniform at about $330^{\circ} \mathrm{C}$. The PbLi manifold design is challenging because:

$\square$ it shall withstand the maximum pressure of $18.6 \mathrm{MPa}$, in accidental conditions;

$\square$ it shall be integrated with the feeding and draining pipes of the PbLi loop systems and the BZ, ensuring that the overall pressure drops of the PbLi system are reasonably low (i.e. $<2.0 \mathrm{MPa}$ ); $\square$ considering the significant reduction of space in radial direction of DEMO2017, it shall contribute to the TBR performances;

$\square \quad$ it shall ensure a uniform PbLi distribution in toroidal direction and an efficient recirculation in the BZ;

$\square$ potential risks for $\mathrm{PbLi}$ water contact[21] shall be minimized and limited to the rupture of the DWT.

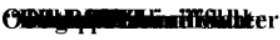

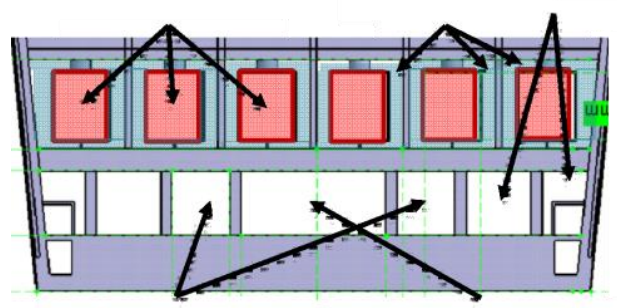

Fig. 1. WCLL 2018 inboard segment: layout of the BSS, water and $\mathrm{PbLi}$ manifolds.

One of the main concerns for the feasibility of the $\mathrm{PbLi}$ manifold and its integration approach is the MHD pressure loss. To achieve an acceptable value, the PbLi flow path in the blanket is designed to maintain throughout a relatively low velocity (e.g. $13 \mathrm{~mm} / \mathrm{s}$ in the outer manifold and $0.01 \mathrm{~mm} / \mathrm{s}$ in the breeding zone). The WCLL outboard blanket is taken as reference and the pressure loss therein is estimated according to literature correlations for laminar, inertia-less, inviscid MHD pressure-driven flow.. The MHD pressure drop has two main contributions: the term $2 \mathrm{D}$ accounting for the losses due to the cross-sectional currents encountered, evaluated according with Ref. [22], and the term 3D [23] representing the losses due to streamwise currents, featured in complex geometrical elements and/or nonuniform boundary conditions. The PbLi path is divided in six hydraulic regions and the calculated pressure losses are summarized in Tab. 1. Nearly $52 \%$ of the total pressure loss is localized in the feeding and draining pipe due to the high velocity $(\approx 55 \mathrm{~mm} / \mathrm{s})$ and the electrical contact with a thick, well-conducting wall, (c $\approx 0.156$ ), whereas the breeding zone contribution is negligible.

Table 1. MHD pressure losses in the PbLi hydraulic path

\begin{tabular}{llc}
\hline$\#$ & Hydraulic Path & Pressure drop $[\mathrm{kPa}]$ \\
\hline 1 & Feeding Pipe & 253 \\
2 & Inlet Manifold & 89 \\
3 & Spinal Channel & 251 \\
4 & Breeding Zone & 15 \\
5 & Outlet Manifold & 111 \\
6 & Draining Pipe & 253 \\
\hline & Outboard Total & 972 \\
\hline
\end{tabular}

\subsection{The breeding and FW zones}

The breeding zone is reinforced by stiffening plates in order to safely withstand the thermo-mechanical and electro-magnetic loads postulated in normal, off normal and accidental conditions. The stiffening approach constrains the Pbli flow path, as well as affects the DWT 
layout. Reliable parametric analyses, supporting the optimization of the plates thickness, imply the enhancement of the TBR performances. In the SMS approach, the study of the stiffening performances has to distinguish between the upper and lower caps and the other zones. In this last case, the analyses reported in Ref. [13] demonstrates that the configuration with the vertical and the horizontal plates having a thickness of $12 \mathrm{~mm}$ and $10 \mathrm{~mm}$, respectively, has a minimum margin of RCCMRx design rules equal to $12.5 \%$. It is highlighted that this configuration has a reduction of the steel in the breeding zone equal to $11.3 \%$, if compared with the configuration WCLL 2016, see also Ref. [24].

The breeding zone cooling approach relies on a breeding cell repeated along the poloidal direction. The main design features and constraints are hereafter synthetized.

$\square$ The coolant flows in DWT, having inner and outer diameters equal to $8 \mathrm{~mm}$ and $13.5 \mathrm{~mm}$, respectively.

$\square$ The main DWT manufacturing requirements are: maximum curvature radius 3 times the external diameter; the minimum distance between the tube axes $26 \mathrm{~mm}$; and the number of curvatures limited to 2.

$\square \quad$ The DWT layout shall ensure that the temperature of the structural material is below the limit, thus $550^{\circ} \mathrm{C}$; shall provide a symmetric thermal field in toroidal direction; shall be compatible with the manifold approach; and shall not cross the stiffening plates in the BZ.

The reference configuration WCLL 2018 (Fig. 2) is based on 20 DWT. The approach fulfils the design requirements above, including, on the opposite of WCLL 2016, the manufacturing requirements and the temperature field symmetry. Moreover, the tube layout provides an overall pipe length reduction of about $25 \%$ and a reduction of steel in the first $175 \mathrm{~mm}$ of BZ of about $50 \%$. Preliminary thermal-hydraulic analyses [16] are carried out using ANSYS CFX 18.1 modelling the coolant, the $\mathrm{PbLi}$, the EUROFER and the tungsten. No hot spot, exceeding the limit, is calculated (Fig. 3), although in the poloidal direction the buoyancy forces are suppressed and only inertial forces are considered (conservative assumption). Efforts are in progress to calculate the thermal and the flow fields through the MHD analysis.

The First Wall integrated in the breeding blanket is in charge of: 1) removing the surface loads; 2) ensuring the structural integrity of the breeding zone; 3 ) delivering the coolant to the FW Primary Heat Transfer System heat exchanger for an efficient power conversion into electricity. On the plasma facing area, the FW is coated by the tungsten layer of $2 \mathrm{~mm}$. The square cooling channels have a side equal to $7 \mathrm{~mm}$ and are placed with the horizontal axes at $6.5 \mathrm{~mm}$ from the FW surface. With the pitch of $13.5 \mathrm{~mm}$, the geometry is optimized [25] in order to achieve the best compromise between thermalhydraulic and thermo-mechanics performances. System [26] and CFD [27] code analyses have demonstrated that, if the FW heat flux exceed about $1.34 \mathrm{MW} / \mathrm{m}^{2}$, the integrated $\mathrm{FW}$ shall be protected with a dedicated detached system. Moreover, when this heat load is below $0.4 \mathrm{MW} / \mathrm{m}^{2}$, it is possible to increase the pitch up to 22.5 $\mathrm{mm}$, without relevant variations of the temperature distribution of the metallic structure, i.e. the maximum temperature remains below $450{ }^{\circ} \mathrm{C}$. This outcome implies that having a reliable and accurate thermal loads of the FW in all operative conditions, it is possible to choose the appropriate pitch (larger than $13.5 \mathrm{~mm}$ ) in order to maximize the thermo-mechanical and TBR performances, minimizing the amount of water [28].

Finally, it must be stressed that the evaluation of the DNB condition has demonstrated that the boiling crisis is excluded.

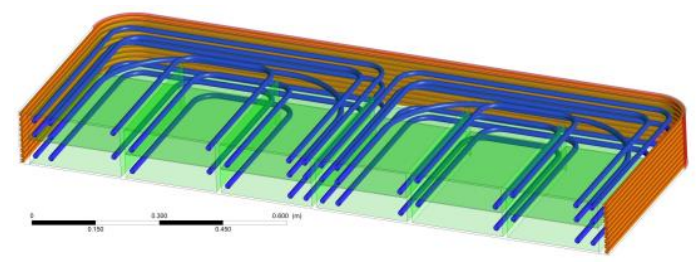

Fig. 2. WCLL 2018 OB equatorial breeding cell geometry.

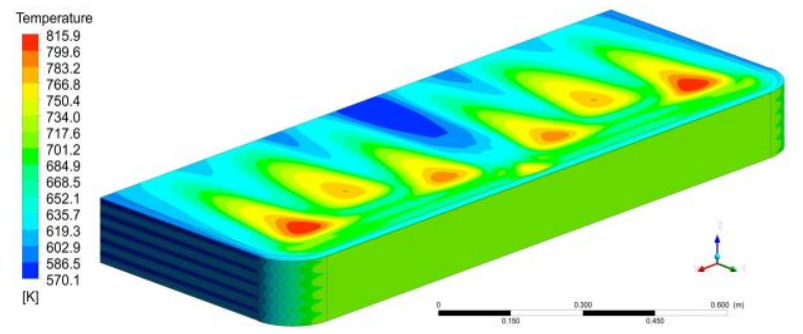

Fig. 3. WCLL 2018 OB equatorial breeding cell: Eurofer and Tungsten domain temperature

\section{Integration with the PbLi loop}

The PbLi loop [6] is aimed at circulating the PbLi through the blanket at about $328^{\circ} \mathrm{C}$, carrying the $\mathrm{T}$ generated in the BB (about 270-280g/day) towards the Tritium Extraction and Removal System (TER), contributing in the $\mathrm{T}$ extraction, controlling $\mathrm{PbLi}$ chemistry, purifying the $\mathrm{PbLi}$ from the impurities. In general, it is designed to ensure a reliable and safe operation of the system. The total amount of $\mathrm{PbLi}$ is about $1.000 \mathrm{~m}^{3}$ divided into six loops. This is stored in dedicated tanks when DEMO power plant is in shutdown conditions. These tanks are placed in the lower part of the circuit to facilitate the drainage by gravity.

Each PbLi loop (Fig. 4) is composed by a charge tank, a storage tank, an expansion/He relief tank, a pumping system, a TER, a heat exchanger and a purification system. The integration of the PbLi loop with the BB is designed by means of the upper port for the loops connected with the outboard segments. On the opposite, the connections with the inboard segments are through the lower port for the inlet and the upper port for the outlet. This is connected with the different mass flow rates of these loops, which imply a diameter of the outboard PbLi pipes equal DN200. This dimension is not compatible with the space reservation in the lower port of the Vacuum Vessel (currently equal to DN80). It should be noted that 
the connecting $\mathrm{PbLi}$ pipe diameter is driven mainly by the pressure drops connected with the presence of the magnetic field, sect. 2.1.

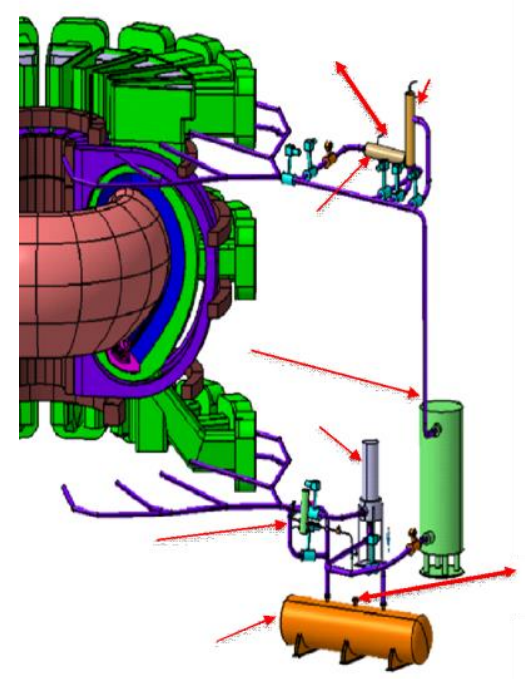

Fig. 4. Layout of a PbLi loop for WCLL BB inboard segments.

\section{Integration with the PHTS and CPS}

The water-cooled Primary Heat Transfer System (PHTS) is constituted by two independent systems (Fig. 5): the BZ PHTS and FW PHTS [29]. The BB water manifolds are connected with the sector collectors and distributors with pipelines routed through the upper port. The largest inlet and outlet pipes are DN200 and are connected with the BZ system of the outboard segment. In general, the pipe diameter is chosen minimizing the diameter size, but accounting for the maximum velocity limit of $7 \mathrm{~m} / \mathrm{s}$ for the pipelines in EUROFER inside the bio-shield.

During the pulse mode the BZ-PHTS delivers the power $\left(1483.2 \mathrm{MW}_{\text {th }}\right)$ to the steam turbine by means of two Once Through Steam Generators (OTSG). The steam is super-heated $\left(300.0^{\circ} \mathrm{C}\right)$ at the pressure of $6.4 \mathrm{MPa}$. The thermal power transported by the FW-PHTS (439.8 $\left.\mathrm{MW}_{\text {th }}\right)$ is exchanged through two horizontal Intermediate Heat Exchangers, tubes and shell type, to an Intermediate Heat Transfer System (IHTS) using HITEC molten salt as coolant. The reference configuration assumes the temperature cycle $295.0-328.0{ }^{\circ} \mathrm{C}$ for the primary water coolant system and $280.0-320.0{ }^{\circ} \mathrm{C}$ for the intermediate molten salt coolant system. The IHTS exchanges heat power to the PCS by means of 4 Helical Coil Steam Generators. These are operated for delivering superheated steam at the nominal conditions (i.e. 6.4 MPa, $300.0^{\circ} \mathrm{C}$ ). The overall pumping power of the PHTSs are 4.2 MW and 13.5 MW, for the FW and the BZ main coolant pumps, respectively. During pulse, 173.9 $\mathrm{MW}_{\text {th }}$ out of $439.8 \mathrm{MW}_{\text {th }}$ are stored in the ESS, whereas the remaining are bypassed towards the PCS, thus the steam turbine. When the plasma is off, the ESS, consisting into two tanks of about $11000 \mathrm{~m}^{3}$, feeds the PCS with the thermal energy needed to keep the PCS feed water components on and the turbine at the nominal power. The analyses on the proposed configuration [30] showed that the water cooled DEMO power plant, operated in pulse mode, can provide a constant gross electrical power of about $760 \mathrm{MW}$, with a gross efficiency of $36 \%$.

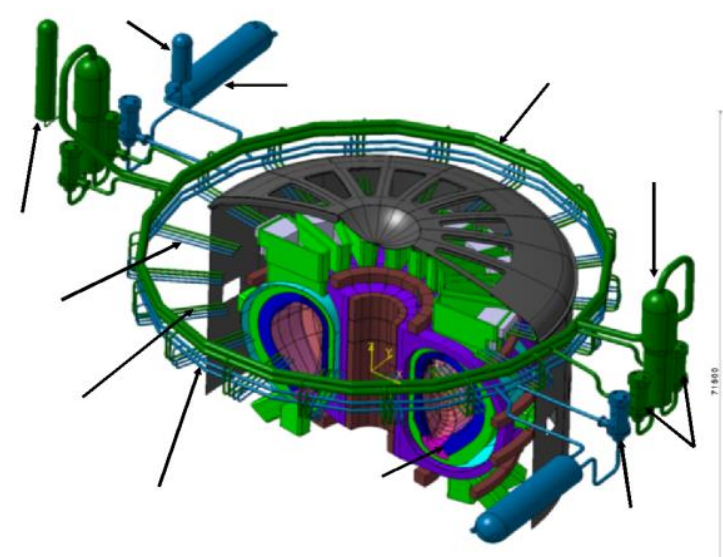

Fig. 5. Layout of the FW and BZ PHTS.

\section{Conclusive remarks}

The preliminary conceptual design of the WCLL BB is up-dated at the version 2018, accounting for the availability of the new DEMO 2017 baseline, characterized by a remarkable radial reduction of the $\mathrm{BB}$ space. Cornerstones are single module segment approach and the water manifold between the breeding blanket box and the back supporting structure.

The integration of the water coolant systems is defined. On the opposite the integration of the PbLi systems is proposed and shall be further investigated evaluating if the He is always dissolved in the liquid metal and exploiting if the electrical insulation of inlet and outlet pipes can be considered.

WCLL BB 2018 is characterized by a meaningful steel reduction, derived from the thermo-mechanics and thermos-hydraulics studies (i.e. stiffeners and tubes). Moreover, the studies on the FW have confirmed the cooling limit, but also the margin available for adapting the channel pitch to the thermal load specifications. Having reliable and accurate thermal loads of the FW in all operative conditions, this will allow to enhance both the thermo-mechanical and the TBR performances. Current reference design will be further studied and optimized for the pre-Conceptual Design Review planned in 2020. Moreover, R\&D programs are ongoing and in tight connection with the design activities. Relevant samples are: the manufacturing studies; the quantification of the $\mathrm{T}$ permeation in the cooling system and of the $\mathrm{He}$ bubble formation in the BZ; and the demonstration that the pressure, temperature and $\mathrm{H}_{2}$ formation during "inbox-LOCA" does not compromise the structural integrity of the BB box.

\section{Acknowledgments}

This work has been carried out within the framework of the EUROfusion Consortium and has received funding from the Euratom research and training programme 20142018 under grant agreement No 633053. The views and 
opinions expressed herein do not necessarily reflect those of the European Commission.

\section{References}

[1] L.V. Boccaccini et al., Objectives and status of EUROfusion DEMO blanket studies, Fusion Eng. Des., 109-111 (2016), pp. 1199-1206.

[2] G. Federici et al., An Overview of the EU Breeding Blanket Design Strategy as Integral Part of the DEMO Design Effort, Proceedings of the $30^{\text {th }}$ SOFT, Italy, 2018.

[3] A. Del Nevo et. al., WCLL breeding blanket design and integration for DEMO 2015: status and perspectives, Fusion Eng. Des., 124 (2017), pp. 682-686.

[4] E. Martelli et. al., Advancements in DEMO WCLL breeding blanket design and integration, Int. J. of Energy Research, 42(1), 2018, pp. 27-52.

[5] A. Tassone et al., Recent Progress in the WCLL Breeding Blanket Design for the DEMO Fusion Reactor, IEEE Transactions on Plasma Science, 46 (5), 2018, pp. 14461457.

[6] M. Utili et al. Development of Pb-16Li technologies for DEMO Reactor, Proceedings of the $30^{\text {th }}$ SOFT, Italy, 2018.

[7] F. Romanelli et al., Fusion Electricity - A roadmap to the realization of fusion energy, EFDA (Nov., 2012), pp. 2028 ISBN 978-3-00-0407.

[8] L. Barucca et al, Status of EU DEMO heat transport and power conversion systems, Fusion Eng. Des., 2018 DOI: 10.1016/j.fusengdes.2018.05.057.

[9] F. Moro et al., Neutronic analyses in support of the WCLL DEMO design development, Fusion Eng. Des., 2018 DOI: 10.1016/j.fusengdes.2018.04.113.

[10] E. Martelli et al., Thermal-hydraulic modeling and analyses of the water-cooled EU DEMO using RELAP5 system code, Proceedings of the $30^{\text {th }}$ SOFT, Italy, 2018.

[11] G. Federici et al., DEMO design activity in Europe: Progress and updates, Fusion Eng. Des., 2018 DOI: 10.1016/j.fusengdes.2018.04.001.

[12] G. Bongiovì et al., Multi-Module vs. Single-Module concept: Comparison of thermomechanical performances for the DEMO Water-Cooled Lithium Lead breeding blanket, Fusion Eng. Des., 2018 DOI: 10.1016/j.fusengdes.2018.05.037.

[13] P.A. Di Maio et al., On the effect of stiffening plates configuration on the DEMO Water Cooled Lithium Lead Breeding Blanket module thermo-mechanical behavior, Proceedings of the 30th SOFT, Italy, 2018.

[14] R. Mozzillo et al., Alternative design of DEMO Water Cooled Lithium Lead internal structure, Proceedings of the $30^{\text {th }}$ SOFT, Italy, 2018.

[15] R. Forte et al., On the effects of the Double-Walled Tubes lay-out on the DEMO WCLL breeding blanket module thermal behaviour, Proceedings of the $30^{\text {th }}$ SOFT, Italy, 2018.

[16] F. Edemetti et al., EU DEMO WCLL BB breeding zone cooling system design: analysis and discussion, Proceedings of the $30^{\text {th }}$ SOFT, Italy, 2018.

[17] A. Tassone et al., MHD mixed convection flow in the WCLL: heat transfer analysis and cooling system optimization, Proceedings of the 30th SOFT, Italy, 2018.

[18] M. Kordac et al., Helium bubble formation in Pb-16Li within the breeding blanket, Fusion Eng. Des., 124 (2018), pp. 700-704.

[19] P.A. Di Maio et al., Structural analysis of the back supporting structure of the DEMO WCLL outboard blanket, Fusion Eng. Des., 124 (2017), pp. 944-947

[20] I. A. Maione et al., Analysis of EM loads on DEMO WCLL breeding blanket during VDE-up, Fusion Eng.
Des., DOI: 10.1016/j.fusengdes.2018.05.048.

[21] M. Eboli et al., Simulation study of pressure trends in the case of loss of coolant accident in Water Cooled Lithium Lead blanket module, Fusion Eng. Des., 98-99 (2015), pp. 1763-1766.

[22] I.R. Kirillov et al., Present understanding of MHD and heat transfer phenomena for liquid metal blankets, Fusion Eng. Des. 27 (1995), pp. 553-569.

[23] S. Smolentsev et al, MHD thermofluid issues of liquidmetal blankets: Phenomena and advances, Fusion Eng. Des. 85 (2010), pp. 1196-1205.

[24] P.A. Di Maio, et. al., On the thermo-mechanical behaviour of DEMO water-cooled lithium lead equatorial outboard blanket module, Fusion Eng. Des., 124 (2017), pp. 725729.

[25] P. A. Di Maio et al., On the optimization of the first wall of the DEMO water-cooled lithium lead outboard breeding blanket equatorial module, Fusion Eng. Des., 109-111 (2016), pp. 335-341.

[26] A. Froio et al., Parametric thermal-hydraulic analysis of the EU DEMO Water-Cooled Lithium-Lead First Wall using the GETTHEM code, Fusion Eng. Des., 137 (2018), pp. 257-267.

[27] K. Jiang et al., Investigation on cooling performance of WCLL BB FW for EU DEMO, Proceedings of the $30^{\text {th }}$ SOFT, Italy, 2018.

[28] P. Chiovaro et al., Parametric Study of the Influence of First Wall Coolant on the WCLL Breeding Blanket Nuclear Response, Proceedings of the $30^{\text {th }}$ SOFT, Italy, 2018.

[29] E. Martelli et al., Study of EU DEMO WCLL breeding blanket and primary heat transfer system integration, Fusion Eng. Des., 2018 DOI: 10.1016/j.fusengdes.2018.04.016.

[30] L. Malinowski et al., Design and analysis of the improved configuration of the secondary circuit for the EU-DEMO power plant, Proceedings of the $30^{\text {th }}$ SOFT, Italy, 2018. 